\title{
Salivary Glucose Concentration and Excretion in Normal and Diabetic Subjects
}

\author{
Cedric Jurysta, ${ }^{1}$ Nurdan Bulur, ${ }^{1}$ Berrin Oguzhan, ${ }^{1}$ Ilhan Satman, ${ }^{2}$ Temel M. Yilmaz, ${ }^{2}$ \\ Willy J. Malaisse, ${ }^{1}$ and Abdullah Sener ${ }^{1}$ \\ ${ }^{1}$ Laboratory of Experimental Hormonology, Free University of Brussels, B-1070 Brussels, Belgium \\ ${ }^{2}$ Endocrinology Department, Istanbul University Medical School, 34390 Istanbul, Turkey \\ Correspondence should be addressed to Abdullah Sener, abdsener@ulb.ac.be
}

Received 5 February 2009; Accepted 17 March 2009

Recommended by Francesca Cutruzzolà

The present report aims mainly at a reevaluation of salivary glucose concentration and excretion in unstimulated and mechanically stimulated saliva in both normal and diabetic subjects. In normal subjects, a decrease in saliva glucose concentration, an increase in salivary flow, but an unchanged glucose excretion rate were recorded when comparing stimulated saliva to unstimulated saliva. In diabetic patients, an increase in salivary flow with unchanged salivary glucose concentration and glucose excretion rate were observed under the same experimental conditions. Salivary glucose concentration and excretion were much higher in diabetic patients than in control subjects, whether in unstimulated or stimulated saliva. No significant correlation between glycemia and either glucose concentration or glucose excretion rate was found in the diabetic patients, whether in unstimulated or stimulated saliva. In the latter patients, as compared to control subjects, the relative magnitude of the increase in saliva glucose concentration was comparable, however, to that of blood glucose concentration. The relationship between these two variables was also documented in normal subjects and diabetic patients undergoing an oral glucose tolerance test.

Copyright () 2009 Cedric Jurysta et al. This is an open access article distributed under the Creative Commons Attribution License, which permits unrestricted use, distribution, and reproduction in any medium, provided the original work is properly cited.

\section{Introduction}

Many authors found higher glucose salivary levels in diabetic patients than in nondiabetics [1-11]. Such investigations aimed mainly at exploring whether diabetic control could be monitored by a noninvasive method of salivary glucose measurement [1-4]. The latter remains, however, a matter of controversies [5-8]. Several factors may account for the poor correlation between blood and saliva glucose concentrations prevailing in diabetic subjects. They include oral retention of alimentary carbohydrates $[12,13]$, glucose utilization by oral bacteria [14], release of carbohydrates from salivary glycoproteins $[15,16]$, and contamination of saliva by a large outflow of crevicular fluid in patients with a poor gingival status $[17,18]$.

In considering the relationship between salivary glucose concentration and salivary flow, the present study mainly aimed at re-evaluating salivary glucose concentration and excretion in unstimulated and mechanically stimulated saliva in both normal and diabetic subjects.

\section{Materials and Methods}

2.1. Subjects. The present report deals with five sets of experiments. The first set of experiments was conducted in 38 normal subjects, including 16 males and 22 females with respective mean ages $( \pm$ SEM) of $24 \pm 2$ and $23 \pm$ 2 years. The second set of experiments was conducted in 84 diabetic patients, including 36 males and 48 females with mean respective ages of $56 \pm 3$ and $55 \pm 2$ years. The third set of experiments was restricted to 9 normal subjects and 18 diabetic patients. At variance with the first two sets of experiments, it did not include measurements of salivary flow and, hence, glucose excretion rates. The fourth set of experiments consisted of an oral glucose tolerance test conducted in 4 normal subjects and 2 diabetic patients. The last set of experiments concerned 3 healthy subjects and 2 diabetic patients, examined at the occasion of successive samplings of stimulated saliva in the absence of any change in glycemia. The diabetic patients were treated and appropriate control subjects were recruited from 
the Endocrinology Department, Istanbul University Medical School, Istanbul, Turkey, and the Stomatology Department, Erasmus Hospital, Université Libre de Bruxelles, Brussels, Belgium. All experiments and sample collections, as well as saliva glucose measurements, were performed by the same investigator either in Turkey or Belgium.

The present research was conducted in full accordance with ethical principles, including the World Medical Association Declaration of Helsinki.

2.2. Saliva Sample Collection. To collect saliva, a standardized tube with two compartments and a standardized cotton were used. Both the cotton and two-compartment tube were obtained from the same manufacturer (SalivetteTM, Starstedt, Nümbrecht, Germany). The upper part of the tube containing the cotton presented a hole, so that, after centrifugation, the saliva was recovered in the lower part and became available for analysis.

Saliva was collected in fasting subjects, immediately after rinsing the oral cavity two times with $150 \mathrm{~mL}$ of water and drinking this water, by means of cotton kept in the oral cavity for 1 to 3 minutes either in the unstimulated state or during mastication (stimulated saliva). The cotton was transferred in the upper part of the tube. Salivary flow was determined by weighing the device with the cotton before and after saliva collection, assuming that $1 \mathrm{~g}$ of saliva corresponds to $1 \mathrm{~mL}$. Centrifugation of the device at $2000 \mathrm{~g}$ for 5 minutes allowed the saliva adsorbed to the cotton to pass through the orifice into the lower compartment of the device, the saliva being then immediately frozen at $-20^{\circ} \mathrm{C}$.

Although salivary flux could be affected by the use of salivette, the latter was used to standardize the collection of saliva, for hygiene reasons, and to remove particles from the saliva.

Blood was taken from finger tip, and blood glucose concentration was measured by the glucose oxidase method [19].

2.3. Salivary Glucose Assay. Salivary D-glucose concentration was determined by the hexokinase method adapted from [20]. $100 \mu \mathrm{L}$ centrifuged saliva was mixed with $95 \mu \mathrm{L}$ of reagent medium containing $2.0 \mathrm{mM} \mathrm{MgCl} 2,0.5 \mathrm{mM}$ ATP, $0.5 \mathrm{mM} \mathrm{NADP}^{+}$, and 0.06 units of yeast glucose 6-phosphate dehydrogenase in TRIS-HCl buffer $(200 \mathrm{mM}, \mathrm{pH}$ 8.1). After a first reading of the absorbance at $340 \mathrm{~nm}$, the reaction was started by the addition of $5 \mu \mathrm{L}$ yeast hexokinase in reagent medium (0.06 units). The absorbance at $340 \mathrm{~nm}$ was recorded after 30-minutes incubation at room temperature. The assay was simultaneously conducted on glucose standards (final concentration comprised between 5 and $250 \mu \mathrm{M})$. The results were calculated as nmol of glucose/mL saliva after the subtraction of reading in the absence of hexokinase and taking into account glucose standards and saliva volume.

The coefficient of variation is, respectively, $3.3 \pm 0.4 \%$ $(n=25)$ and $5.4 \pm 0.6 \%(n=51)$ for D-glucose standards and saliva samples. The standard curve of glucose between 5 to $250 \mu \mathrm{M}$ is linear with a correlation coefficient of 0.999 .
Our method can measure as little as $0.5 \mathrm{nmol}$ of glucose with a variation coefficient of $4.3 \%$.

2.4. Statistical Analaysis. All results are presented as mean values $( \pm$ SEM $)$ together with the number of individual determinations $(n)$ or degree of freedom (d.f.). The statistical significance of differences between mean values was assessed by the use of Student's $t$-test.

\section{Results}

In a large series, the glucose concentration was $76.4 \pm 3.8$ (111) and $32.4 \pm 2.4 \mu \mathrm{M}$ (126), respectively, in unstimulated and stimulated saliva from normal subjects.

A first study was conducted in 38 normal subjects, including 16 males and 22 females. The glucose concentration averaged $79.4 \pm 5.8 \mu \mathrm{M}(n=33)$ in unstimulated saliva, as distinct $(P<.001)$ from only $32.4 \pm 4.4 \mu \mathrm{M}(n=38)$ in stimulated saliva. The salivary flow increased $(P<.001)$ from a basal value of $0.82 \pm 0.09 \mathrm{~mL} / \mathrm{min}$ to a stimulated value of $1.88 \pm 0.09 \mathrm{~mL} / \mathrm{min}$ ( $n=38$ in both cases). The glucose excretion rate failed, however, to differ significantly $(P>.3)$ in unstimulated saliva $(66.7 \pm 6.1 \mathrm{nmol} / \mathrm{min} ; n=33)$ and stimulated saliva ( $57.1 \pm 8.3 \mathrm{nmol} / \mathrm{min} ; n=38)$, with a mean paired difference between unstimulated and stimulated saliva of $14.1 \pm 11.7 \mathrm{nmol} / \mathrm{min}(n=33 ; P>.2)$. As a rule, these variables did not differ significantly in male versus female subjects (Table 1). The stimulated salivary flow, however, was higher $(P<.01)$ in male than in female subjects.

A comparable study was then conducted in 84 diabetic patients, including 15 subjects with type-1 diabetes ( 6 males and 9 females) and 69 subjects with type- 2 diabetes (30 males and 39 females). The glucose concentration averaged $187.3 \pm 20.0 \mu \mathrm{M}(n=74)$ in unstimulated saliva and $187.5 \pm$ $19.6 \mu \mathrm{M}(n=83)$ in stimulated saliva. The salivary flow increased $(P<.005)$ from a basal value of $1.18 \pm 0.08 \mathrm{~mL} / \mathrm{min}$ to a stimulated value of $1.51 \pm 0.06 \mathrm{~mL} / \mathrm{min}(n=84$ in both cases). The glucose excretion rate, however, failed to differ significantly $(P>.4)$ in the unstimulated saliva $(223.0 \pm 35.6 \mathrm{nmol} / \mathrm{min} ; n=74)$ and stimulated saliva $(255.7 \pm 28.1 \mathrm{nmol} / \mathrm{min} ; n=83)$. None of these variables differed significantly in type- 1 and type- 2 diabetic patients of the same gender. Likewise, the glucose concentration failed to differ significantly in male and female diabetic patients, whether in unstimulated or stimulated saliva. The basal salivary flow and glucose excretion rate were lower $(P<.05$ or less), however, in female diabetic patients than in male diabetic subjects (Table 1). Moreover, a significant increase in salivary flow $(P<.001)$ and glucose excretion rate $(P<.05)$, in response to stimulation, was only observed in the female diabetic patients (Table 1).

The glycemia in the diabetic patients averaged $9.75 \pm$ $0.48 \mathrm{mM}(n=84)$, representing about twice the mean value otherwise found in normal subjects (see below). Likewise, the glucose concentration in unstimulated saliva was about twice higher in the diabetic patients $(187.3 \pm 20.0 \mu \mathrm{M} ; n=$ 74) than in the control subjects $(79.4 \pm 5.8 \mu \mathrm{M} ; n=33)$. In the diabetic patients, as compared to control subjects, 
TABLE 1: Unstimulated and stimulated data in normal and diabetic subjects.

\begin{tabular}{lcccc}
\hline & \multicolumn{2}{c}{ Normal } & \multicolumn{2}{c}{ Diabetic } \\
\hline Gender & Male & Female & Male & Female \\
Glucose concentration $(\mu \mathrm{M})$ & & & & \\
$\quad$ Unstimulated & $78.7 \pm 9.2(14)$ & $80.4 \pm 7.9(19)$ & $201.9 \pm 34.9(34)$ & $175.0 \pm 22.3(40)$ \\
$\quad$ Stimulation & $29.7 \pm 8.1(16)$ & $34.4 \pm 5.0(22)$ & $203.6 \pm 37.2(36)$ & $175.1 \pm 19.9(47)$ \\
$\quad$ Salivary flow ( $\mathrm{mL} / \mathrm{min})$ & & & & \\
$\quad$ Unstimulated & $0.82 \pm 0.17(16)$ & $0.82 \pm 0.11(22)$ & $1.53 \pm 0.13(36)$ & $0.92 \pm 0.09(48)$ \\
$\quad$ Stimulation & $2.17 \pm 0.14(16)$ & $1.67 \pm 0.11(22)$ & $1.63 \pm 0.10(36)$ & $1.42 \pm 0.08(48)$ \\
Glucose excretion (nmol/min) & & & & \\
$\quad$ Unstimulated & $60.9 \pm 7.1(14)$ & $71.3 \pm 9.3(19)$ & $302.0 \pm 72.5(34)$ & $155.7 \pm 19.5(40)$ \\
$\quad$ Stimulation & $59.8 \pm 16.5(16)$ & $55.2 \pm 8.2(22)$ & $291.7 \pm 54.8(36)$ & $228.1 \pm 26.6(47)$ \\
\hline
\end{tabular}

the relative magnitude of the increase in unstimulated saliva glucose concentration thus failed to differ significantly $(P>$ .1) from that in glycemia. The unstimulated saliva flow was also somewhat higher $(P<.01)$ in the diabetic patients $(1.18 \pm 0.08 \mathrm{~mL} / \mathrm{min} ; n=84)$ than in the control subjects $(0.82 \pm 0.09 \mathrm{~mL} / \mathrm{min} ; n=38)$. Hence, the mean basal glucose excretion rate was about thrice higher $(P<.005)$ in diabetic patients $(223.0 \pm 35.6 \mathrm{nmol} / \mathrm{min} ; n=74)$ than in the control subjects $(66.7 \pm 6.1 \mathrm{nmol} / \mathrm{min} ; n=33)$.

In a further set of experiments, the glucose concentration in stimulated saliva was again found to decrease $(P<.005$, paired comparison) from an unstimulated value of $81.0 \pm$ 19.9 to $33.3 \pm 3.9 \mu \mathrm{M}(n=9)$ in healthy subjects, whilst no significant decrease $(P>.2)$ was observed in 18 diabetic patients. In this set of experiments, the glycemia averaged in diabetic patients $8.76 \pm 0.77 \mathrm{mM}(n=17)$ as compared $(P<.005)$ to $4.95 \pm 0.14 \mathrm{mM}(n=8)$ in control subjects, yielding a diabetic/control ratio of $177 \%$. Likewise, the glucose concentration in basal and stimulated saliva averaged in the diabetic patients $179.7 \%$ of the mean corresponding values found in control subjects. In the diabetic patients, as compared to control subjects, the relative magnitude of the increase in saliva glucose concentration was thus, once again, comparable $(P>.9)$ to that of blood glucose concentration, with an overall diabetic/control ratio of $178.8 \pm 24.2 \%(n=$ 53; $P<.025$ versus the reference value of $100.0 \pm 9.8 \%$; $n=28)$.

In order to investigate further the relationship between blood and saliva glucose concentration, an oral glucose tolerance test ( $75 \mathrm{~g}$ ) was conducted in 4 normal subjects and 2 diabetic patients (Figure 1). The glucose concentration in unstimulated saliva progressively increased during the first 30 minutes of the test, reaching a peak value which averaged $253 \pm 49 \%(P<.01)$ of paired basal measurement. Thereafter, the glucose concentration in unstimulated saliva samples progressively decreased, eventually reaching at minutes 120 180 nadir values representing no more than $47.4 \pm 14.8 \%$ $(P<.06)$ of paired basal measurement.

The unexpected fall in saliva glucose concentration below basal value observed during the late part of the oral glucose tolerance test led us to measure such a concentration at the occasion of successive samplings in the absence of change in glycemia. As illustrated in Figure 2, which refers to a study conducted in 3 healthy subjects and 2 diabetic patients from whom 8 successive samples of stimulated saliva were collected over a period of 17 minutes, a progressive fall in glucose concentration was indeed recorded under these experimental conditions.

Figure 3 illustrates the mean values for saliva glucose concentrations, salivary flow, and glucose excretion rate in all unstimulated and stimulated samples collected in this study. It emphasizes the decrease in glucose concentration $(P<.001)$, increase in salivary flow $(P<.001)$, but unchanged glucose excretion rate $(P>.3)$ recorded in normal subjects when comparing stimulated to unstimulated saliva. It also documents the increase in salivary flow $(P<.005)$ with unchanged salivary glucose concentration $(P>.99)$ and glucose excretion rate $(P>.4)$ observed under the same experimental conditions in diabetic patients. Last, it illustrates the marked increase $(P<.001)$ in salivary glucose concentration and glucose excretion rate found in diabetic patients, as compared to normal subjects, whether in unstimulated or stimulated saliva. The latter finding contrasts with more modest differences $(P<.01$ or less) between normal subjects and diabetic patients for unstimulated or stimulated salivary flow, the results collected in the latter patients averaging $112.1 \% \pm 5.7 \%(n=168$; $P>.2$ ) of the corresponding mean values recorded under the same experimental conditions in normal subjects $(100.0 \% \pm$ $5.9 \% ; n=76)$.

Despite the high number of individual determinations, no significant correlation was found in the diabetic patients between glycemia and either glucose concentration $(r=$ 0.1614 ; d.f. $=91 ; P>.1)$ or glucose excretion rate $(r=$ -0.0629 ; d.f. $=72 ; P>.1$ ) in unstimulated saliva. Likewise, no significant correlation could be found in the diabetic patients between glycemia and either glucose concentration $(r=0.1545$; d.f. $=98 ; P>.1)$ or glucose excretion rate $(r=0.1522$; d.f. $=81 ; P>.1)$ in stimulated saliva.

\section{Discussion}

In the present study, the unstimulated salivary flow was higher compared to the unstimulated saliva flow of about 


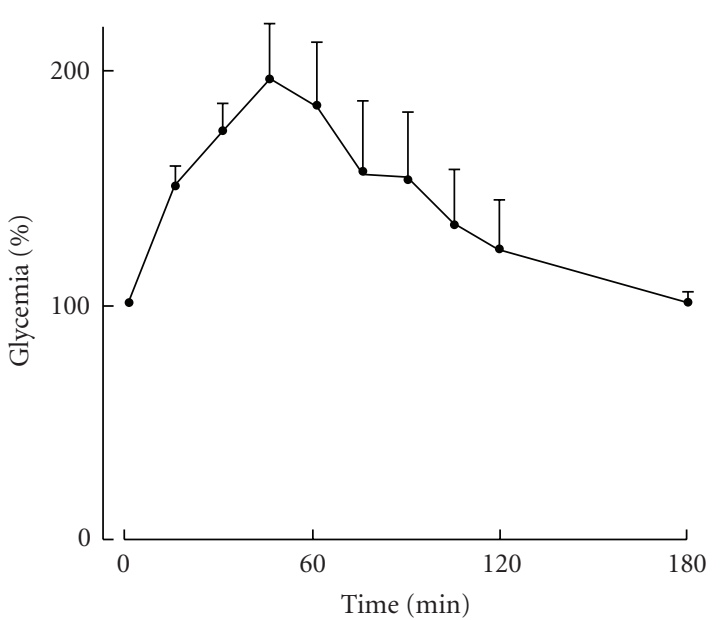

(a)

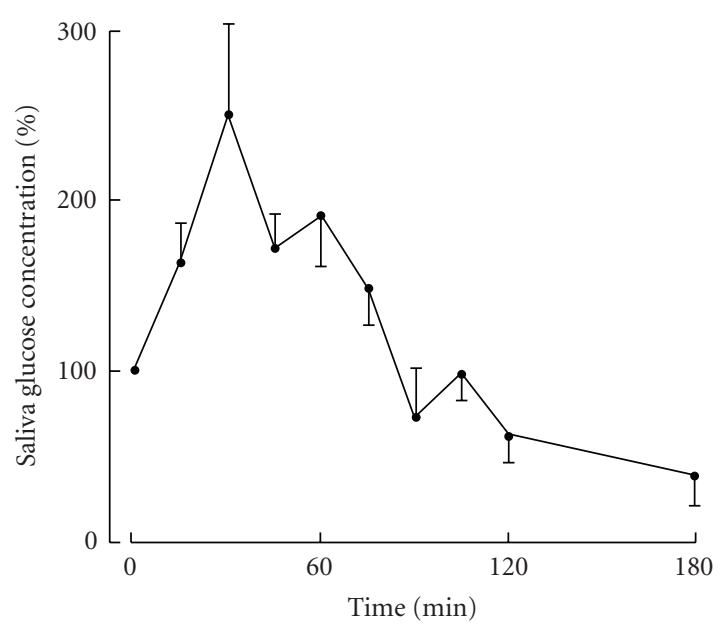

(b)

FIGURE 1: Time course for the changes in glycemia and unstimulated saliva glucose concentration in 4 normal subjects and 2 diabetic patients during an oral glucose tolerance test. Geometric mean values $( \pm$ SEM) refer to results expressed in percent of paired basal value.

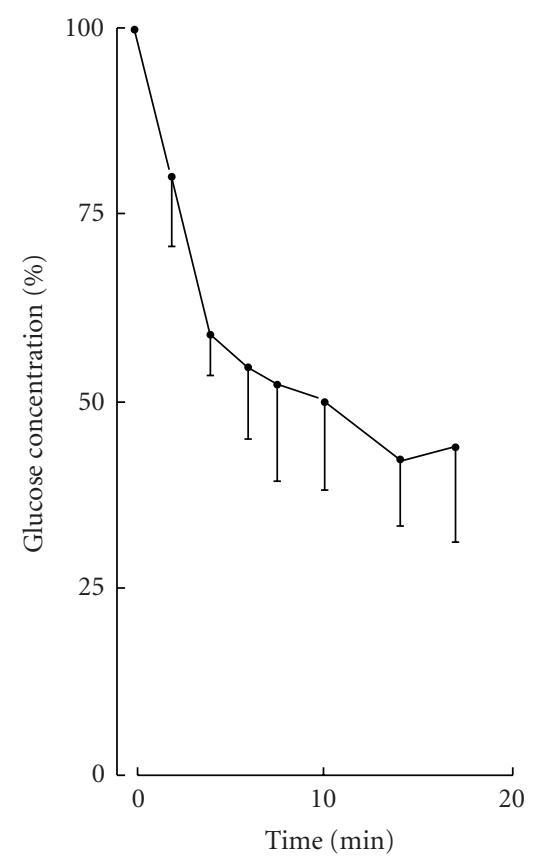

Figure 2: Time course for the changes in glucose concentration during successive samplings of stimulated saliva in 5 subjects. Geometric mean values ( \pm SEM) refer to results expressed in percent of paired first measurement.

$0.4 \mathrm{~mL} / \mathrm{min}$ observed in many studies [21-26]. This situation is probably linked to the use of a salivette for the collection of saliva.

The present results confirm that the glucose concentration in saliva is higher in diabetic patients than in control subjects [1-11]. It extends this knowledge to both unstimulated and stimulated saliva. It also confirms that, in both normal subjects and diabetic patients, the salivary flow is higher in stimulated as compared to unstimulated saliva
[7, 21-28]. Despite such an increase, the glucose excretion rate, taken as the product of saliva glucose concentration multiplied by salivary flow, failed to differ significantly under unstimulated and stimulated conditions, whether in normal subjects or diabetic patients. The latter finding argues in support of a dissociated regulation of salivary flow (increased by mechanical stimulation) and glucose release by salivary glands (unaffected by mechanical stimulation).

The dependency of saliva glucose concentration on glycaemia was further documented by the time course of changes in the former variable during an oral glucose tolerance test, as documented in both normal subjects and diabetic patients. During the glucose tolerance test (OGTT), the salivary glucose level increased twofold within 60 minutes, as observed previously $[29,30]$. The measurements of saliva glucose concentrations made during such an oral glucose tolerance test led us to observe, in a further set of experiments, that such a concentration decreases at the occasion of successive samplings of stimulated saliva, such a decrease occurred despite unchanged salivary flow. Its pattern was reminiscent of the rapid clearance of exogenous glucose from the saliva of human subjects otherwise observed during the first 6 to 8 minutes, followed by a much slower clearance thereafter [30-32].

No significant difference between type- 1 and type-2 diabetic subjects was detected in the present study, and no significant correlation between glycemia and glucose saliva concentration or glucose excretion rate was found in the diabetic patients, whether in unstimulated or stimulated saliva. These findings confirm the poor link between glycaemia and glucose concentration or excretion in saliva, at least on an individual basis [5-8]. Nevertheless, the present study may well set the scene for further investigations on the regulation of glucose output from salivary glands, as well as on the potentially unfavorable effect of a high glucose salivary concentration on selected variables of oral health status in diabetic patients. 

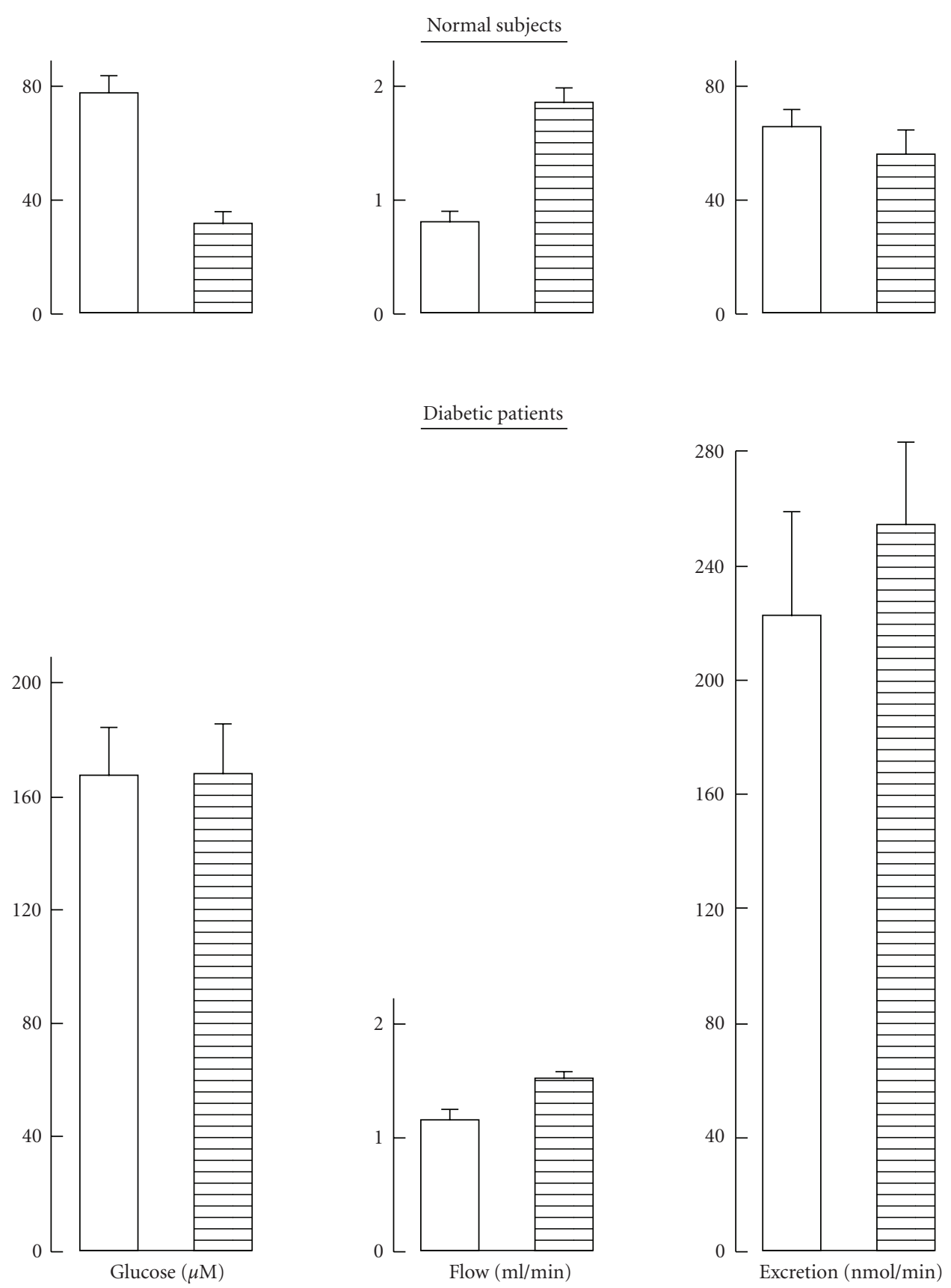

FIGURE 3: Glucose concentration, salivary flow, and glucose excretion rate for unstimulated (open columns) and stimulated (hatched columns) saliva in normal subjects and diabetic patients. Mean values $( \pm$ SEM) are derived from all data collected in the present study and, hence, refer to 33-101 individual measurements.

\section{Acknowledgment}

This study was supported by the Belgian Foundation for Scientific Medical Research (Grant 3.4520.07).

\section{References}

[1] K. K. Mehrotra, T. N. Chawla, and A. Kumar, "Correlation of salivary sugar with blood sugar," Journal of the Indian Dental Association, vol. 40, no. 10, pp. 265-269, 1968.
[2] C. O. Reuterving, G. Reuterving, E. Hägg, and T. Ericson, "Salivary flow rate and salivary glucose concentration in patients with diabetes mellitus influence of severity of diabetes," Diabète et Métabolisme, vol. 13, no. 4, pp. 457-462, 1987.

[3] P. Marchetti, M. Tognarelli, R. Giannarelli, et al., "Decreased salivary glucose secretory rate: usefulness for detection of diabetic patients with autonomic neuropathy," Diabetes Research and Clinical Practice, vol. 7, no. 3, pp. 181-186, 1989.

[4] S. Amer, M. Yousuf, P. Q. Siddqiui, and J. Alam, "Salivary glucose concentrations in patients with diabetes mellitus- a 
minimally invasive technique for monitoring blood glucose levels," Pakistan Journal of Pharmaceutical Sciences, vol. 14, no. 1, pp. 33-37, 2001.

[5] L. N. Forbat, R. E. Collins, G. K. Maskell, and P. H. Sönksen, "Glucose concentrations in parotid fluid and venous blood of patients attending a diabetic clinic," Journal of the Royal Society of Medicine, vol. 74, no. 10, pp. 725-728, 1981.

[6] H. Ben-Aryeh, M. Cohen, Y. Kanter, R. Szargel, and D. Laufer, "Salivary composition in diabetic patients," Journal of Diabetic Complications, vol. 2, no. 2, pp. 96-99, 1988.

[7] K. M. Karjalainen, M. L. Knuuttila, and M. L. Käär, "Salivary factors in children and adolescents with insulin-dependent diabetes mellitus," Pediatric Dentistry, vol. 18, no. 4, pp. 306311, 1996.

[8] E. M. Chávez, L. N. Borrell, G. W. Taylor, and J. A. Ship, "A longitudinal analysis of salivary flow in control subjects and older adults with type 2 diabetes," Oral Surgery, Oral Medicine, Oral Pathology, Oral Radiology, and Endodontics, vol. 91, no. 2, pp. 166-173, 2001.

[9] A. M. G. Darwazeh, T. W. MacFarlane, A. McCuish, and P.-J. Lamey, "Mixed salivary glucose levels and candidal carriage in patients with diabetes mellitus," Journal of Oral Pathology \& Medicine, vol. 20, no. 6, pp. 280-283, 1991.

[10] M. E. López, M. E. Colloca, R. G. Páez, J. N. Schallmach, M. A. Koss, and A. Chervonagura, "Salivary characteristics of diabetic children," Brazilian Dental Journal, vol. 14, no. 1, pp. 26-31, 2003.

[11] S. Aydin, "A comparison of ghrelin, glucose, alpha-amylase and protein levels in saliva from diabetics," Journal of Biochemistry and Molecular Biology, vol. 40, no. 1, pp. 29-35, 2007.

[12] J. C. Hase and D. Birkhed, "Oral sugar clearance in elderly people with prosthodontic reconstructions," Scandinavian Journal of Dental Research, vol. 99, no. 4, pp. 333-339, 1991.

[13] J. C. Hase, D. Birkhed, F. Lagerlöf, and E. Thornqvist, "Oral retention of glucose at pharmacologically reduced salivary flow in man," Scandinavian Journal of Dental Research, vol. 102, no. 3, pp. 180-185, 1994.

[14] M. H. de Jong, J. S. van der Hoeven, J. H. van OS, and J. H. Olijve, "Growth of oral Streptococcus species and Actinomyces viscosus in human saliva," Applied and Environmental Microbiology, vol. 47, no. 5, pp. 901-904, 1984.

[15] S. A. Leach and P. Critchley, "Bacterial degradation of glycoprotein sugars in human saliva," Nature, vol. 299, no. 5022, p. 506, 1966.

[16] S. A. Leach and T. H. Melville, "Investigation of some human oral organisms capable of releasing the carbohydrates from salivary glycoproteins," Archives of Oral Biology, vol. 15, no. 1, pp. 87-88, 1970.

[17] O. Kjellman, "The presence of glucose in gingival exudate and resting saliva of subjects with insulin-treated diabetes mellitus," Svensk Tandläkare Tidskrift, vol. 63, no. 1, pp. 1119, 1970.

[18] A. J. Ficara, M. P. Levin, M. F. Grower, and G. D. Kramer, "A comparison of the glucose and protein content of gingival fluid from diabetics and nondiabetics," Journal of Periodontal Research, vol. 10, no. 3, pp. 171-175, 1975.

[19] H. U. Bergmeyer and E. Bernt, "Determination with glucose oxidase and peroxidase," in Methods of Enzymatic Analysis, $\mathrm{H}$. U. Bergmeyer, Ed., vol. 3, pp. 1205-1215, Academic Press, New York, NY, USA, 1974.

[20] O. H. Lowry and J. V. Passonneau, A Flexible System of Enzymatic Analysis, Academic Press, New York, NY, USA, 1972.
[21] I. L. Shannon, R. P. Suddick, and F. J. Dowd Jr., "Saliva: composition and secretion," Monographs in Oral Science, vol. 2, pp. 1-103, 1974.

[22] U. Heintze, D. Birkhed, and H. Björn, "Secretion rate and buffer effect of resting and stimulated whole saliva as a function of age and sex," Swedish Dental Journal, vol. 7, no. 6, pp. 227-238, 1983.

[23] J. C. Atkinson, "The role of salivary measurements in the diagnosis of salivary autoimmune diseases," Annals of the New York Academy of Sciences, vol. 694, pp. 238-251, 1993.

[24] I. L. Shannon and W. J. Frome, "Enhancement of salivary flow rate and buffering capacity," Journal of the Canadian Dental Association, vol. 39, no. 3, pp. 177-181, 1973.

[25] U. Heintze, D. Birkhed, and H. Björn, "Secretion rate and buffer effect of resting and stimulated whole saliva as a function of age and sex," Swedish Dental Journal, vol. 7, no. 6, pp. 227-238, 1983.

[26] H. Ben-Aryeh, D. Miron, R. Szargel, and D. Gutman, "Wholesaliva secretion rates in old and young healthy subjects," Journal of Dental Research, vol. 63, no. 9, pp. 1147-1148, 1984.

[27] J. H. Meurman, H.-L. Collin, L. Niskanen, et al., "Saliva in non-insulin-dependent diabetic patients and control subjects: the role of the autonomic nervous system," Oral Surgery, Oral Medicine, Oral Pathology, Oral Radiology, and Endodontics, vol. 86, no. 1, pp. 69-76, 1998.

[28] A. D. Mata, D. Marques, S. Rocha, et al., "Effects of diabetes mellitus on salivary secretion and its composition in the human," Molecular and Cellular Biochemistry, vol. 261, no. 1, pp. 137-142, 2004.

[29] A. Borg and D. Birkhed, "Secretion of glucose in human parotid saliva after carbohydrate intake," Scandinavian Journal of Dental Research, vol. 96, no. 6, pp. 551-556, 1988.

[30] A. B. Andersson, D. Birkhed, K. Berntorp, F. Lindgärde, and L. Matsson, "Glucose concentration in parotid saliva after glucose/food intake in individuals with glucose intolerance and diabetes mellitus," European Journal of Oral Sciences, vol. 106, no. 5, pp. 931-937, 1998.

[31] L. M. Sreebny, R. Chatterjee, and I. Kleinberg, "Clearance of glucose and sucrose from the saliva of human subjects," Archives of Oral Biology, vol. 30, no. 3, pp. 269-274, 1985.

[32] D. Goulet, F. Brudevold, A. Tehrani, and F. Attarzadeh, "Sugar clearance from saliva and intra-oral spaces," Journal of Dental Research, vol. 64, no. 3, pp. 411-415, 1985. 

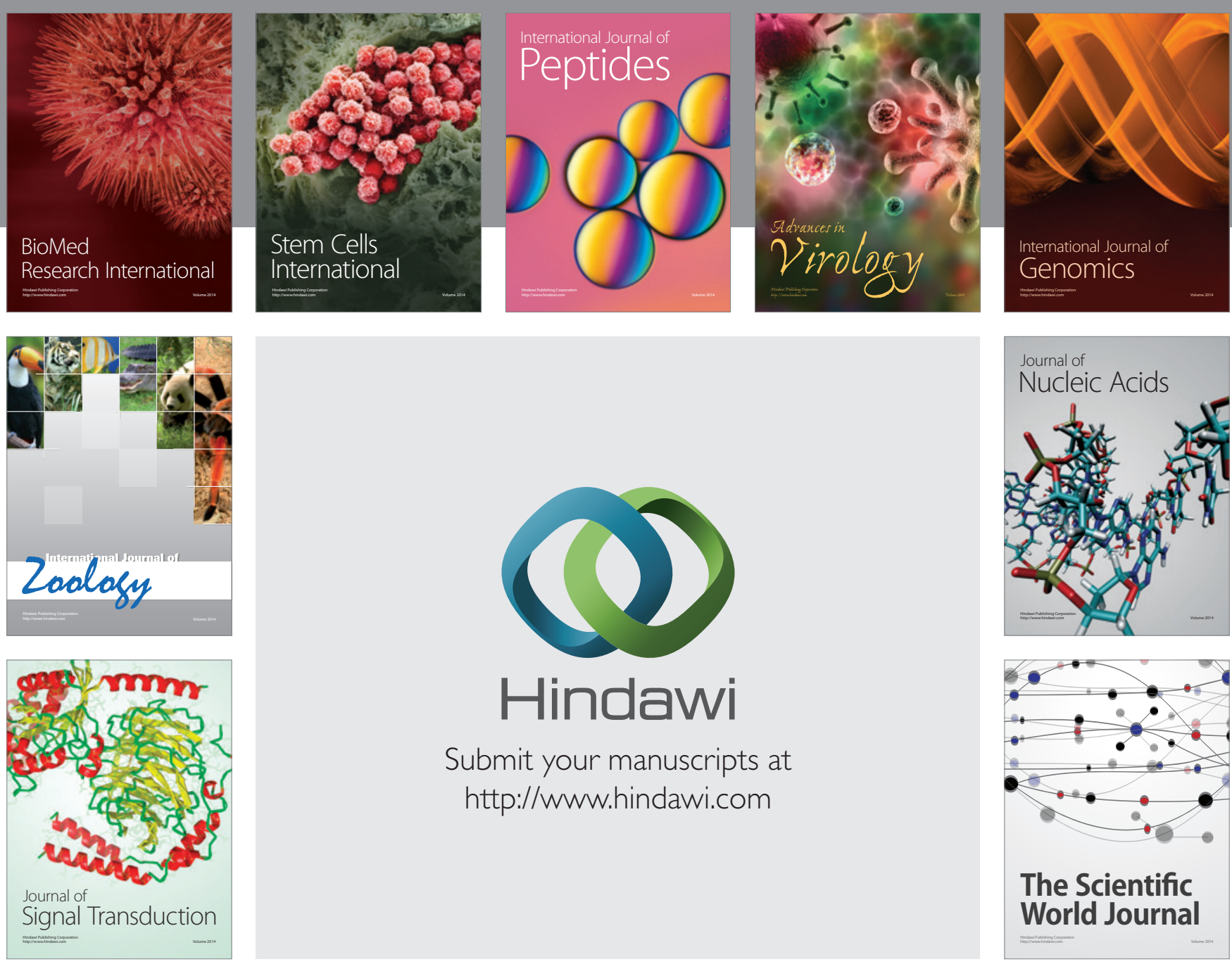

Submit your manuscripts at

http://www.hindawi.com
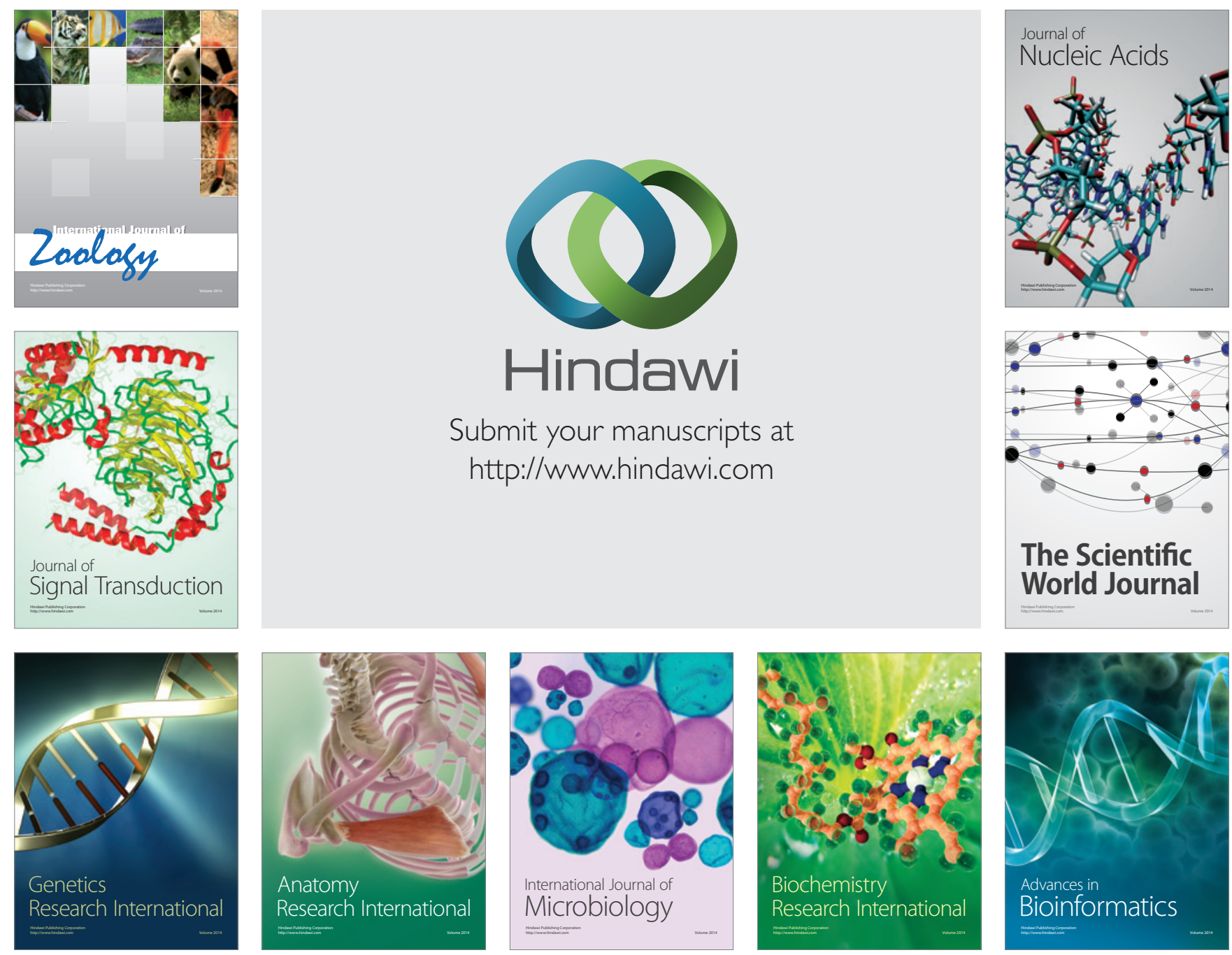

The Scientific World Journal
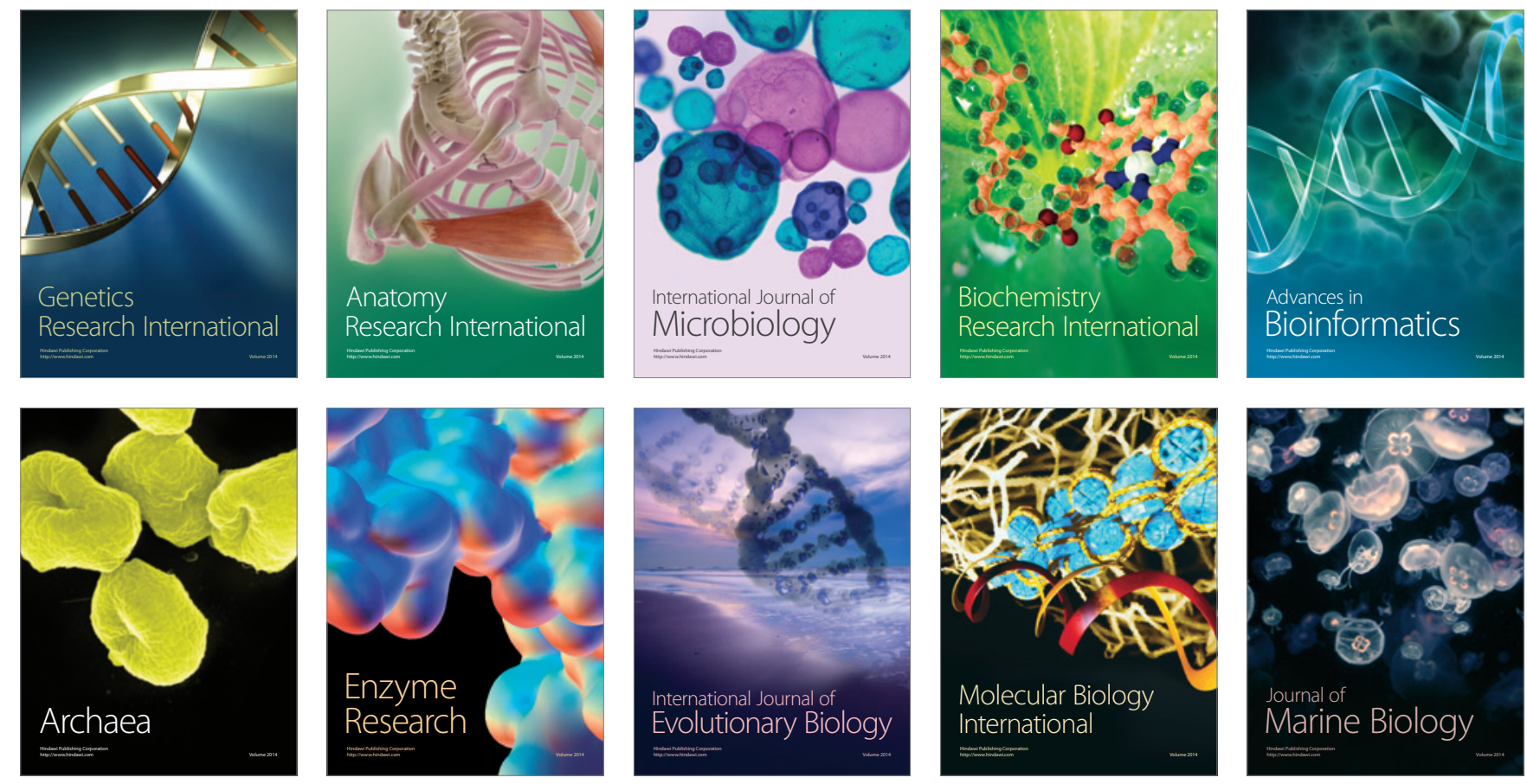